Classification

Physics Abstracts

07.80

\title{
Performance of a new high-resolution electron energy-loss spectroscopy microscope
}

\author{
Masami Terauchi $\left({ }^{1}\right)$, Ryuichi Kuzuo $\left({ }^{1}\right)$, Futami Satoh $\left({ }^{1}\right)$, Michiyoshi Tanaka $\left({ }^{1}\right)$, \\ Katsushige Tsuno $\left({ }^{2}\right)$ and Junichi Ohyama $\left({ }^{2}\right)$ \\ ( $\left.{ }^{1}\right)$ Research Institute for Scientific Measurements, Tohoku University, Katahira 2-1-1, Aoba-ku, \\ Sendai 980, Japan \\ $\left(^{2}\right)$ JEOL LTD., Musashino 3-1-2, Akishima, Tokyo 196, Japan
}

(Received October 31, 1990; accepted March 26, 1991)

\begin{abstract}
We have been developing a new instrument for high resolution electron energy-loss spectroscopy (EELS). It is composed of a JEM-1200EX transmission electron microscope - the basic component - and two Wien filters (a monochrometer and an analyzer) with retardation lenses and acceleration lenses. The stigmatic focus has been achieved by a special design of the Wien filter. A spatial resolution of $190 \mathrm{~nm}$, and a momentum resolution $(2 \pi / \lambda)$ of $0.069 \AA^{-1}$ have been obtained. The energy resolution has attained so far to a value of $81 \mathrm{meV}$.
\end{abstract}

\section{Introduction.}

The aim of our instrument for EELS is to investigate the detailed electronic structures and thermal vibrations of materials with an energy resolution of $10 \mathrm{meV}$ at an accelerating voltage of $80 \mathrm{kV}$. Two further aims are to have an electron energy-loss spectra in the image mode from a specimen area of about $100 \mathrm{~nm}$ in diameter, and in the diffraction mode with a momentum resolution of $0.16 \AA^{-1}$, i.e., one twentieth of the 220 diffraction vector in silicon. To get the required performance, our design uses a field emission gun, and a monochrometer and an analyzer each consisting of a Wien filter plus retardation and acceleration lenses. The double Wien filter arrangement has been previously employed by Boersh, Geiger and coworkers $[1,2,3]$ who achieved a resolution of $3 \mathrm{meV}$ at an accelerating voltage of $30 \mathrm{kV}$, but could only obtain spectra from sample areas greater than about $10 \mu \mathrm{m}$ in diameter.

\section{Design and performance of the Wien filter.}

The Wien filter has an electric field $\mathbf{E}$ in the $x$-direction and a magnetic field $\mathbf{B}$ in the $y$-direction. The two fields are perpendicular to the optical axis (the $z$-direction). A straight electron trajectory is obtained when the Wien condition $E=v B$ is satisfied, $v$ being the velocity of electrons $[1,2,3]$. 
The lengths of the electrodes and the magnetic pole faces along the optical axis were chosen to be $4 \mathrm{~cm}$, so as to obtain an energy resolution of $5 \mathrm{meV}$ for an incident beam of $5 \mu \mathrm{m}$ in diameter with an energy of $20 \mathrm{eV}$. The shape of the Wien filter was determined with the help of electron trajectory calculations, which took account of not only the electric and magnetic fields in the filter but also those in the fringing field regions $[4,5]$. Figure 1 schematically shows the arrangement in the $x y$-plane of the electrodes and the magnetic pole faces of the filter. The tilted magnetic pole faces produce a stigmatic focus. By setting both the distance between electrodes and that between magnetic pole faces to the same values of $1 \mathrm{~cm}$, the Wien condition was almost satisfied in the fringing field regions, as well as in the filter [6].

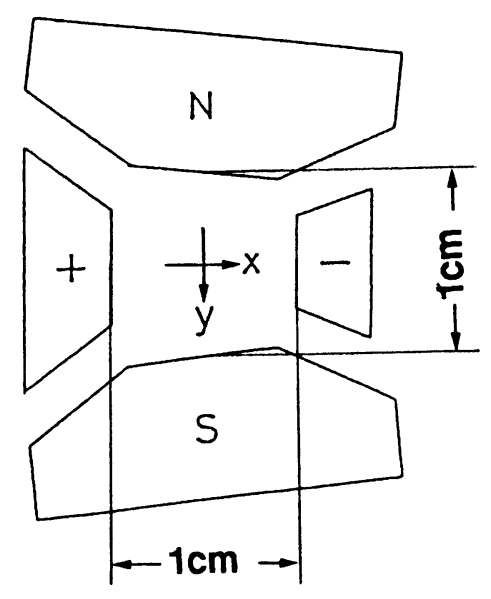

Fig. 1. - Arrangement of the electrodes and the magnetic pole faces in the $x y$-plane of the Wien filter.

Figures 2a to d show a series of beam shapes taken by TV-2 (Fig. 3) at the fluorescent screen behind the analyzer for energy focal lengths of the analyzer $L=2 \pi U_{\mathrm{o}} / v B$ of $124 \mathrm{~mm}(\mathrm{a}), 87 \mathrm{~mm}$ (b), $62 \mathrm{~mm}$ (c) and $50 \mathrm{~mm}$ (d), respectively [7]. $U_{\mathrm{o}}$ represents a retarding potential of the analyzer. The smallest beam of figure $2 \mathrm{c}$ was taken at the stigmatic focus condition, other beam shapes of figures $2 \mathrm{a}, \mathrm{b}$ and $\mathrm{d}$ displaying aberration figures of the filter. The greatest energy dispersion of 3.2 $\mu \mathrm{m} / \mathrm{meV}$ was obtained at $U_{\mathrm{o}}=30 \mathrm{~V}$.

\section{Construction of the instrument.}

Figure 3 shows a schematic diagram of the instrument. It consists of (1) the transmission electron microscope (JEM-1200EX), (2) the monochrometer and the analyzer, (3) the two TV camera systems used for alignment of the electron beam, and (4) the serial detection system of energy loss spectra. The electron microscope is operated at three accelerating voltages (HT) of $40 \mathrm{kV}$, $60 \mathrm{kV}$ and $80 \mathrm{kV}$. The retardation potentials $\left(U_{\mathrm{o}}\right)$ of the Wien filters can be set to an arbitrary value between $20 \mathrm{eV}$ and $2000 \mathrm{eV}$. The monochrometer, the analyzer, and the serial detection system are controlled by a personal computer NEC PC-9801VX [8]. The shape of the electron beam leaving the monochrometer, electron microscope images of specimen and diffraction patterns can be observed by TV -1 . Energy loss spectra produced by the analyzer are observed by TV -2 , which is also used for adjusting the spectrum position. The spectra are recorded by a photomultiplier or a serial detector and are sent to the computer. 


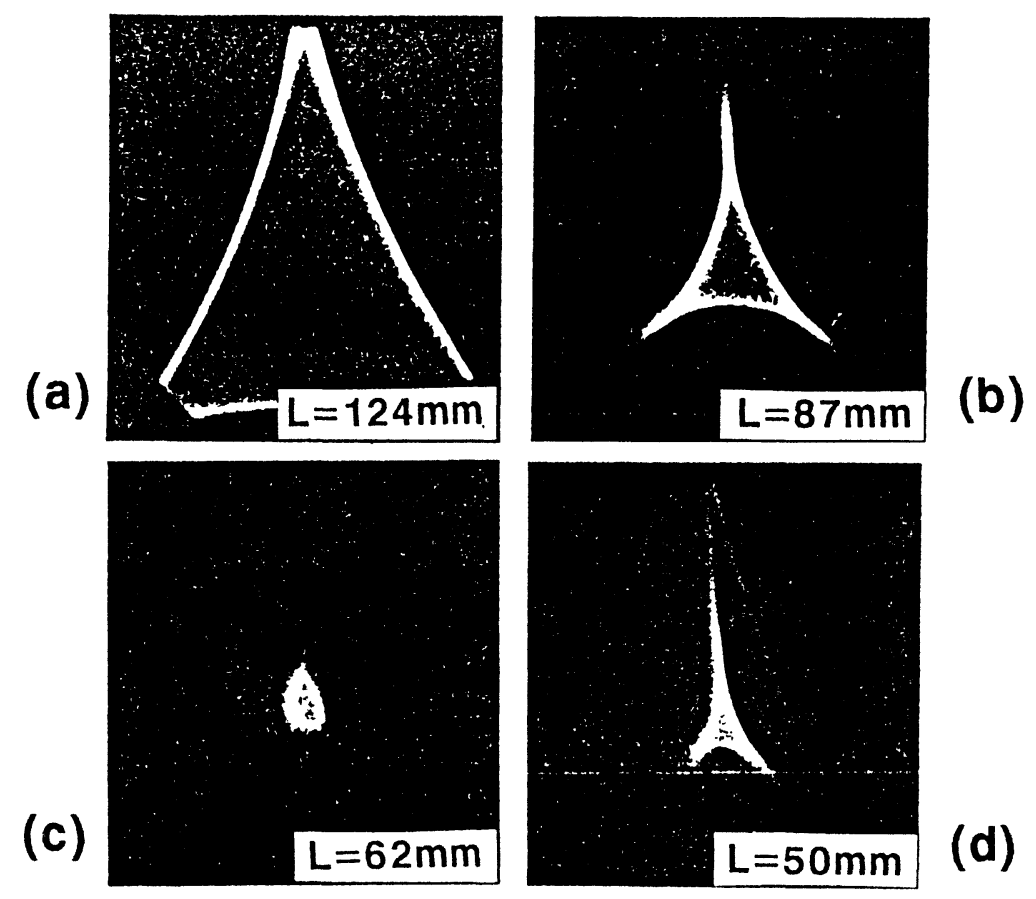

Fig. 2. - A series of beam shapes at the fluorescent screen behind the analyzer as a function of the focal length $L$ of the analyzer.

For energy analysis in the image mode a specimen area is selected by the SA aperture, as in the case of usual electron microscopy [9]. In the diffraction mode a small area in reciprocal space is selected with the same aperture by focusing the diffraction pattern at the aperture position using the objective mini lens (OM in Fig. 3). To obtain the $q$-dependence of the energy loss spectra, the diffraction pattern is shifted by the deflector located at the same height as the objective mini lens, $q$ being the wave-vector.

\section{Application data.}

Figure 4 shows the energy spread of the zero-loss electron beam passing through the monochrometer operated at $U_{\mathrm{o}}=40 \mathrm{~V}$. The distance between the two peaks corresponds to an energy difference of $0.5 \mathrm{eV}$, which was obtained by changing the retarding potential of the analyzer. The full width at half-maximum (FWHM) of the peak was $81 \mathrm{meV}$. Figure 5 shows the spectrum of graphite obtained in the image mode from a specimen area of $190 \mathrm{~nm}$ in diameter with a detection time of $600 \mathrm{~s}$ at a retarding potential of the monochrometer and analyzer of $U_{\mathrm{o}}=40 \mathrm{~V}$. The FWHM of the zero-loss peak obtained was $0.12 \mathrm{eV}$. The energy-loss peaks at about $7 \mathrm{eV}$ and $27 \mathrm{eV}$ are assigned to plasmon losses due to $\pi$-electrons and $(\pi+\sigma)$-electrons, respectively. Figure 6 shows two energy-loss spectra of aluminum obtained in the diffraction mode from a specimen area of $2 \mu \mathrm{m}$ in diameter and from $\Delta q=0.088 \AA^{-1}$ diameter areas in reciprocal space at an HT of $60 \mathrm{kV}$ with a detection time of $200 \mathrm{~s}$. The spectra of figures $6 \mathrm{a}$ and $\mathrm{b}$ were obtained at $q=0$ (i.e., with the direct beam), and at $q=0.302 \AA^{-1}$, respectively. The zero-loss peak seen in figure $6 \mathrm{a}$ is not present in figure $6 \mathrm{~b}$. At an HT of $40 \mathrm{kV}$, a better $\Delta q$ of $0.069 \AA^{-1}$ was obtained. By measuring the 


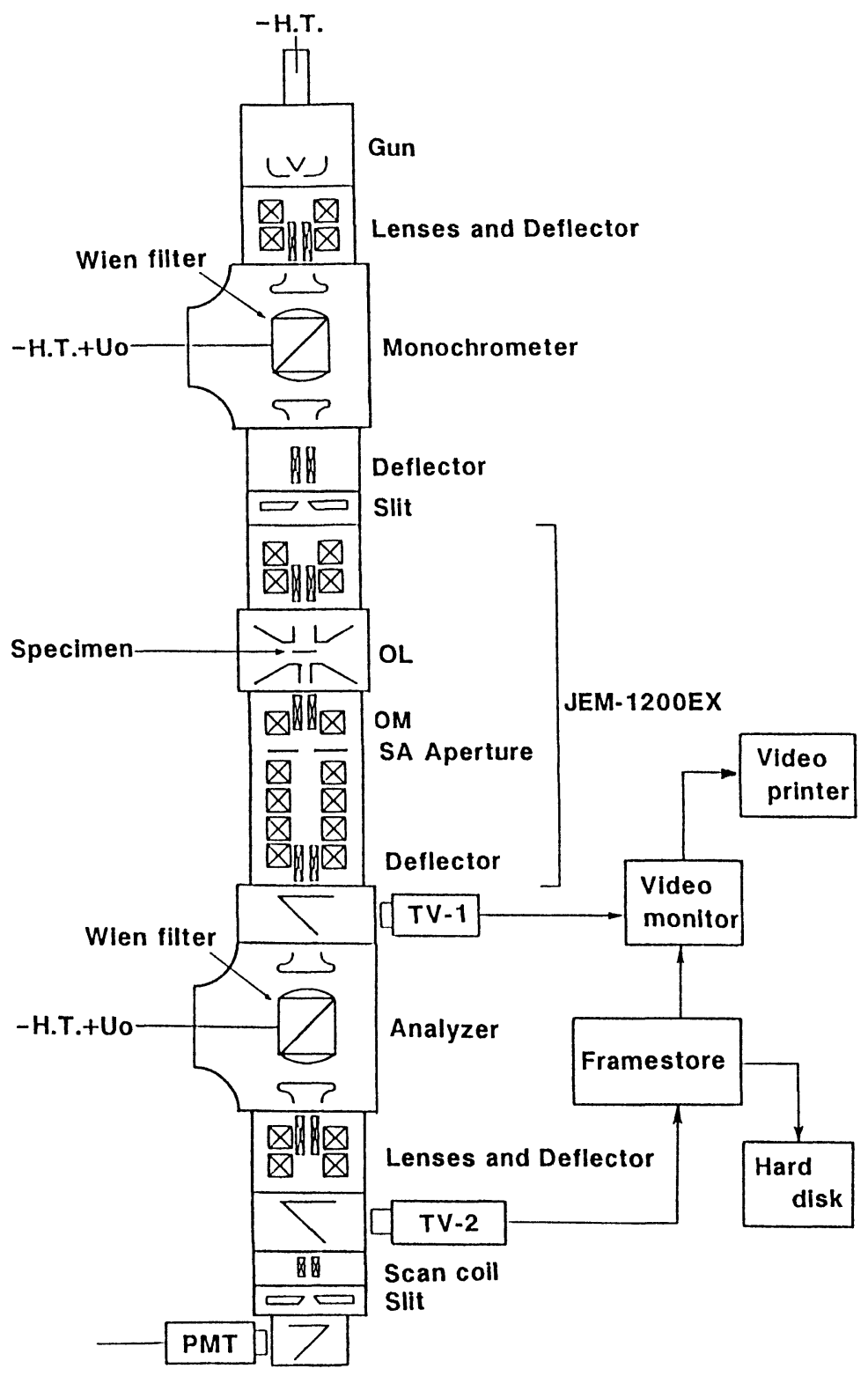

Fig. 3. - Schematic diagram of the instrument.

plasmon loss energy for various values of $q$, the $q^{2}$-dependence of the plasmon energy has been confirmed (Fig. 7). The dots and the inclined straight line in figure 7 represent the experimental results, and the theoretical $q^{2}$-dependence of the plasmon loss energy obtained from the random phase approximation, respectively. As a conclusion, our instrument for high-resolution EELS, at present, has the spatial-resolution of $190 \mathrm{~nm}$, momentum-resolution of $0.069 \AA^{-1}$ and energyresolution of $\sim 0.1 \mathrm{eV}$. To improve the energy resolution, a field emission gun instead of the $\mathrm{LaB}_{6}$ gun and a better Wien filter will be installed. 


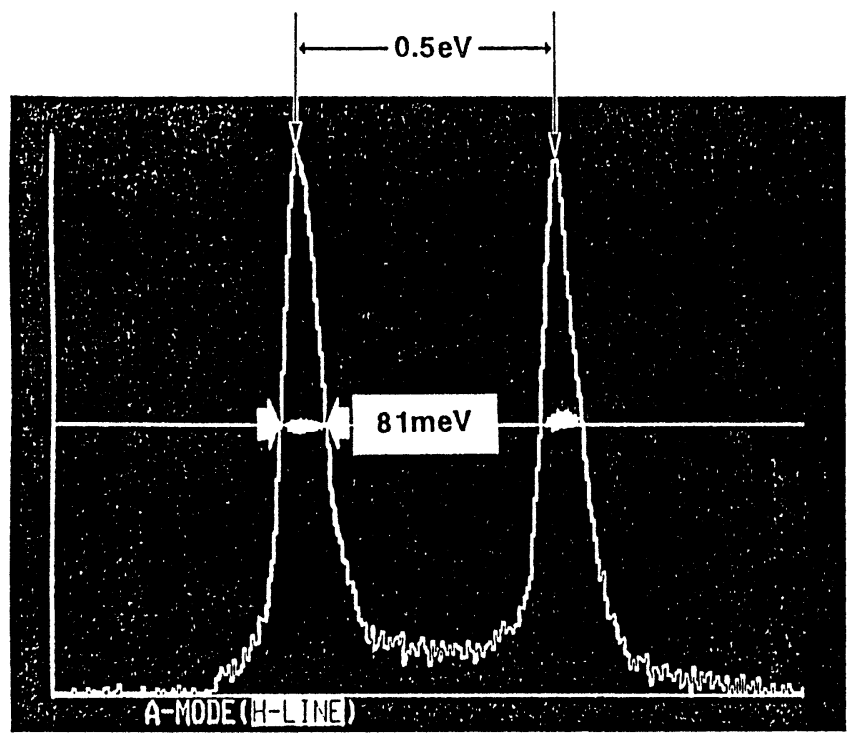

Fig. 4. - Energy spread of electron beams monochromatized by the monochrometer operated at $U_{\mathrm{o}}=$ $40 \mathrm{~V}$.

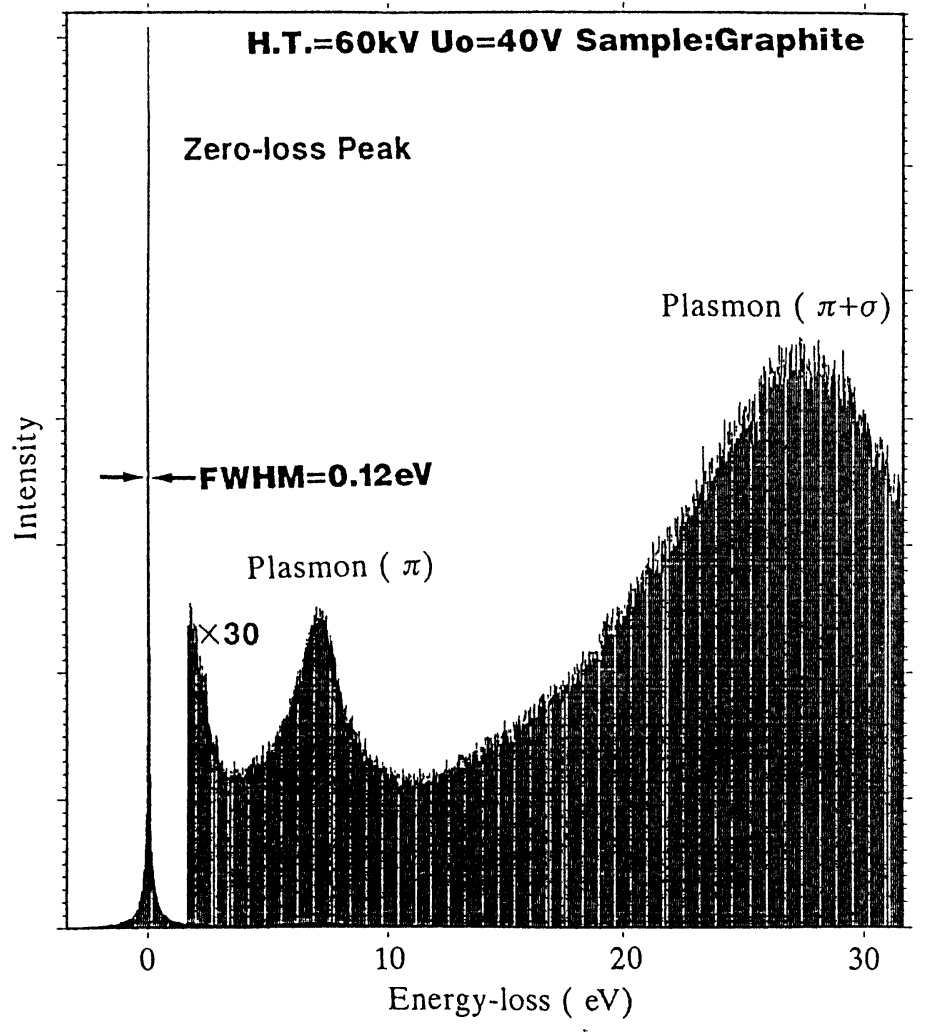

Fig. 5. - Energy loss spectrum of graphite obtained from a specimen area of $190 \mathrm{~nm}$ in diameter in the image mode. 


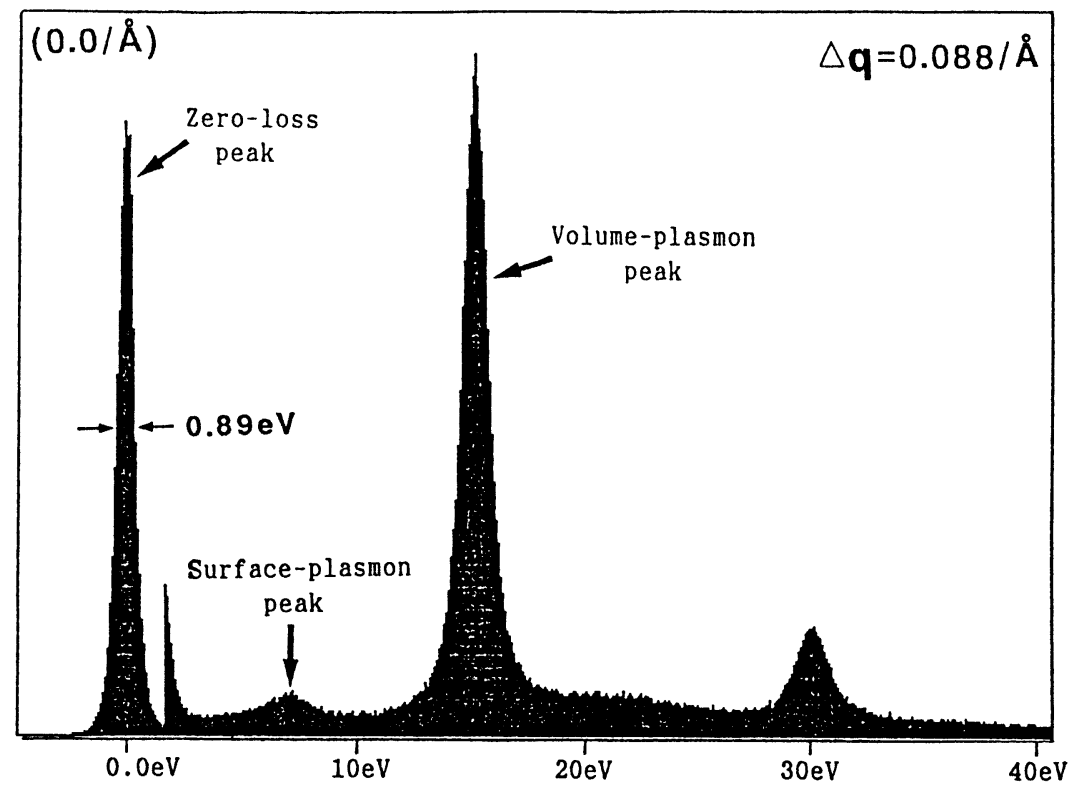

(a)

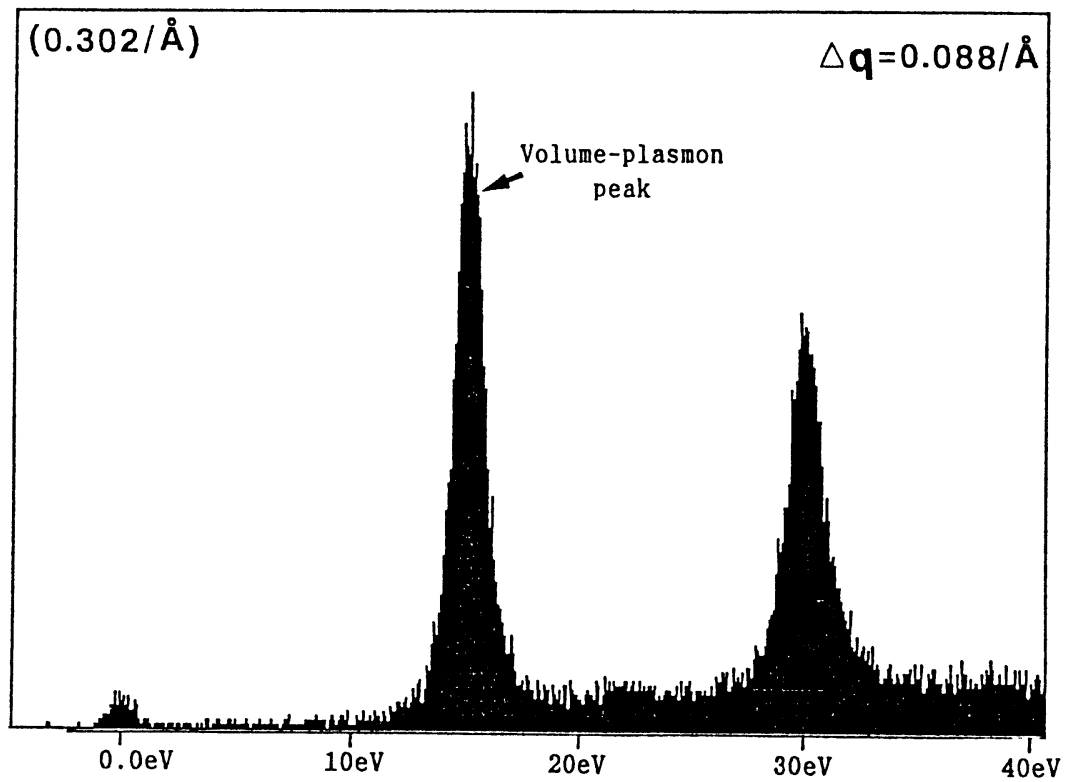

(b)

Fig. 6. - Energy loss spectra of aluminum obtained (a) at $q=0$ (the direct beam), and (b) at $q=0.302 \AA^{-1}$ in the diffraction mode. 


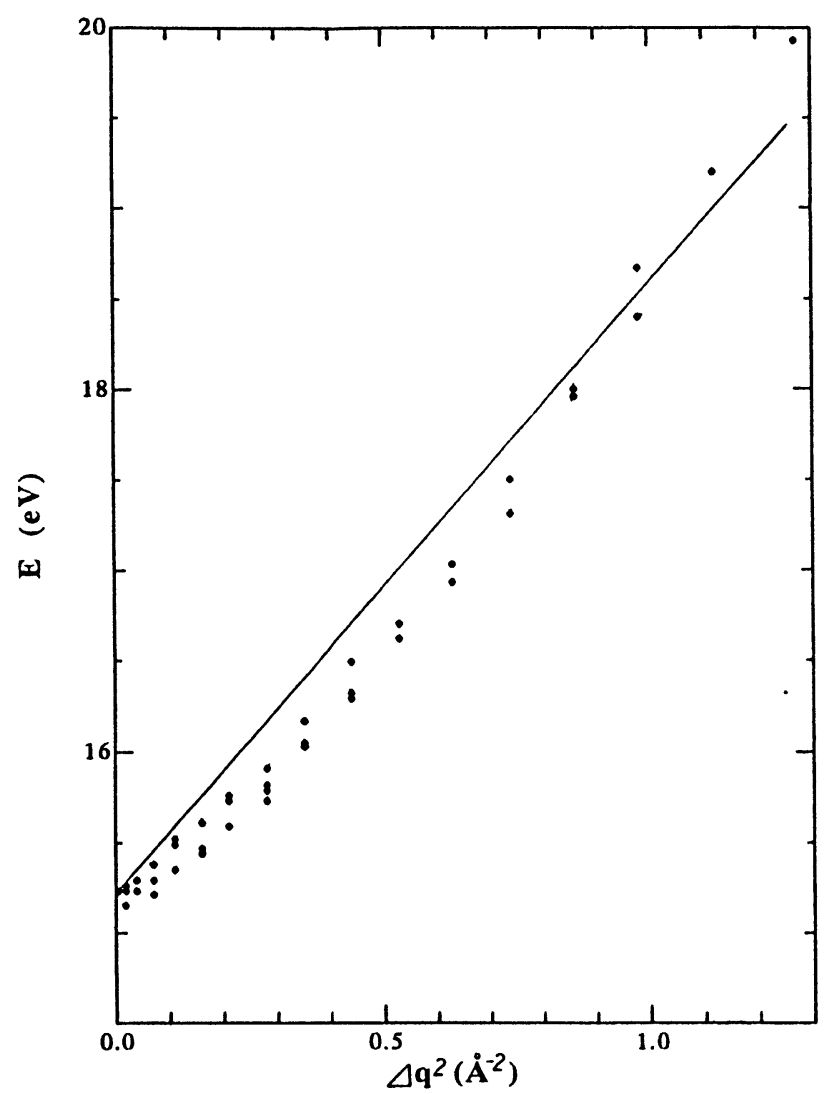

Fig. 7. - The $q^{2}$-dependence of the plasmon loss energy of aluminum. The dots and the inclined straight line represent experimental results, and the $q^{2}$-dependence of the plasmon loss energy obtained from the random phase approximation, respectively.

\section{Acknowledgements.}

The authors would like to thank Dr. M. Essig of BASF Aktiengesellschaft for disclosing his experience with the Wien filter. The present work is supported as a project of Joint Research with Industry by the Ministry of Education, Science and Culture, Japan.

\section{References}

[1] Boersch H., Geiger J. and Stickel W., Z. Phys. 180 (1964) 415.

[2] SCHroder B. and GeIGER J., Phys. Rev. Lett. 28 (1972) 301.

[3] Essig M., Dissertation of the Universitat Kaiserslautern (1981).

[4] Tsuno M., TERAUChI M. and TANAKA M., Optik 78 (1988) 71.

[5] Tsuno M., Terauchi M. and TANaKa M., Optik 80 (1989) 149.

[6] Tsuno M., Terauchi M. and TanaKa M., Inst. Phys. Ser. 98 (1989) 71. 
[7] Terauchi M., Kuzuo R., Satoh F., Tanaka M., Tsuno K. and Ohyama J., Proc. X II th Congr. for Electron Microsc., Seattle (1990) p. 88.

[8] Tsuno K., Ohyama J., Kato M., Kimura J., Kai M., Nakanishi K., Terauchi M. and Tanaka M., Proc. X II th Congr. for Electron Microsc., Seattle (1990) p. 32.

[9] Tsuno M., Terauchi M. and TANaKa M., Optik 83 (1989) 77. 